\title{
Thermomagnetic Convection of Paramagnetic Gas in an Enclosure under No Gravity Condition
}

\author{
Kewei Song ${ }^{1, *}$, Shuai $\mathrm{Wu}^{1}$, Toshio Tagawa ${ }^{2}$, Weina Shi ${ }^{1}$ and Shuyun Zhao ${ }^{3}$ \\ 1 School of Mechanical Engineering, Lanzhou Jiaotong University, Lanzhou 730070, China; \\ m18772101614@163.com (S.W.); pearl_swn@163.com (W.S.) \\ 2 Department of Aeronautics and Astronautics, Tokyo Metropolitan University, Tokyo 191-0065, Japan; \\ tagawa-toshio@tmu.ac.jp \\ 3 Grain Inspection Detachment, Lanzhou Grain Bureau, Lanzhou 730030, China; zhs1266@126.com \\ * Correspondence: songkw@mail.lzjtu.cn
}

Received: 13 December 2018; Accepted: 10 March 2019; Published: 15 March 2019

\begin{abstract}
The thermomagnetic convection of paramagnetic gaseous oxygen in an enclosure under a magnetic field was numerically studied to simulate the thermomagnetic convection in a space environment with no gravity. The magnetic field in the enclosure was non-uniform and was generated by a permanent magnet which had a high magnetic energy product. The magnet was placed at different locations along one of the adiabatic walls with magnetic poles perpendicular to the hot and cold walls of the enclosure. The heat transfer performance, flow field, and temperature field were studied with each location of the magnet. The results show that the thermomagnetic convection in the enclosure was obviously affected by the location of the magnet. There was an optimum magnet location in terms of the best heat transfer performance in the enclosure. The optimum magnet location changed slightly and moved toward the hot wall as the magnetic flux density increased. The value of the Nusselt number, defined as the ratio of convection to conduction, reached up to 2.54 in the studied range of parameters. By optimizing the magnet location, the convection was enhanced by up to $77 \%$ at the optimum magnet location.
\end{abstract}

Keywords: thermomagnetic convection; non-uniform; paramagnetic gas; permanent magnet

\section{Introduction}

Natural convection plays an important role in energy transportation in an enclosure and has been widely studied due to its significant practical applications [1], such as electric equipment cooling, solar energy collection [2], melting and solidification [3], and thermal convection in spacecraft [4]. As the magnetic field can exert a body force on the magnetic fluid, it is possible to control the fluid motion by using a magnetic field [5]. Krakov and Nikiforov [6,7] numerically demonstrated that heat transfer inside an enclosure can be enhanced by increasing the uniform magnetic field. Tangthieng et al. [8] reported an enhancement of the Nusselt number as high as $45 \%$ by applying a permanent magnet on the top of the studied square enclosure. Yu et al. [9] and Tagawa et al. [10] reported that the thermomagnetic convection in a rectangular cavity was obviously affected by the direction of the magnetic field. Vatani et al. [11] studied the thermomagnetic convection of a ferrofluid flow induced by the internal magnetic field around a vertical current-carrying wire and found that increasing the current increased the Nusselt number nonlinearly, ultimately enhancing the heat transfer capability of the induced ferrofluid flow. Vatani et al. [12] experimentally investigated the thermomagnetic convection in ferrofluid in a vertical transient hot-wire cell. They observed that thermomagnetic convection in ferrofluid occurs earlier than natural convection in non-magnetic fluids for similar experimental conditions, and that the onset of thermomagnetic convection was dependent on the 
current supplied to the wire. Jiang et al. [13] numerically investigated the effect of permanent magnetic quadrupole fields on the thermomagnetic convection of air in a porous square enclosure in the presence or absence of a gravity field. Two cellular structures with horizontal symmetry about the middle plane of the enclosure were observed. In addition, the Nusselt number was increased with the increase of the magnetic force number under non-gravitational conditions. Yang et al. [14] also pointed out that the centrifugal-form magnetic force exhibits different flow and heat transfer characteristics from the gravitational free convection. Song et al. [15] numerically studied the effects of magnet position and magnet strength on the thermomagnetic convection in a square enclosure under combined magnetic and gravitational fields. The Nusselt number increased with the increase of magnetic flux density when the magnet was located near a hot wall. The optimum position of a magnet for the best heat transfer is reported. Ashouri et al. [16] numerically studied the pure magnetic convection ferrofluid flow in a square cavity under the magnetic field supplied by a permanent magnet below the bottom wall of the square cavity. Results show that the convective flow and heat transfer rise, increasing with the strengthening of the magnetic field in the absence of a gravity field. Szabo and Früh [17] numerically studied the transition from natural convection to thermomagnetic convection of a magnetic fluid in a non-uniform magnetic field. Their results show that the transition from a single buoyancy-driven convection cell to a single thermomagnetically driven cell is via a two-cell structure, and that the local effect on the flow field leads to a global effect on the heat transfer with a minimum of the Nusselt number in the transition region.

From the above mentioned review we found that most of the published papers focus on the effect of magnetic field direction or the inclined angle of a magnetic field on thermal convection. The thermomagnetic convection in the pure non-uniform magnetic field without a gravity field, such as the requirement in astronautics engineering, has scarcely been studied. The effect of the magnetic field, especially the non-uniform magnetic field supplied by a permanent magnet on thermomagnetic convection is still not well illustrated in the literature. In this paper, the thermomagnetic convection of paramagnetic gaseous oxygen under a pure non-uniform magnetic field supplied by a permanent magnet with magnetic poles paralleling to the adiabatic walls was numerically studied. The location of the magnet was optimized in terms of the strongest thermomagnetic convection in the enclosure.

\section{Physical Model}

The studied physical model is shown schematically in Figure 1. The left wall of the square enclosure was kept at a constant high temperature and named the hot wall. Meanwhile, the right wall of the square enclosure was kept at a constant low temperature and named the cold wall. The other walls, that is, the horizontal walls, were adiabatic. The permanent magnet, the Neodymium-Iron-Boron, which possesses a high residual magnetic flux density, was placed aside the bottom wall, with the middle plane overlapping the square enclosure. The magnet was assumed to be uniformly magnetized in an x-direction, with the magnetic poles parallel to the bottom wall of the enclosure. The length of the square enclosure was $L$ $=0.05 \mathrm{~m}$. The length of the permanent magnet was set to $0.4 \mathrm{~L}$. The gap between the magnet and the enclosure was $\delta=0.04 \mathrm{~L}$. The distance of the magnet from the hot wall was changeable along the bottom wall of the enclosure. The distance between the center of the magnet and the hot wall of the enclosure ranged between $x_{\mathrm{m}} / L=0.0$ and 1.0. Gaseous oxygen, which has a high mass magnetic susceptibility, was considered to be the working medium in the enclosure. The details of the physical properties of gaseous oxygen and other parameters are summarized in Table 1 . The residual magnetic flux density changed from 0.5 Tesla to 3.0 Tesla with an interval of 0.5 Tesla. There were a total of 78 cases with different magnet flux densities and different magnet locations.

The magnetic field lines in the enclosure are shown in Figure 2 for different positions of the magnet. The magnets were placed at $x_{\mathrm{m}} / L=0.2,0.5$, and 0.8 for Figure $2 \mathrm{a}-\mathrm{c}$, respectively. The magnetic fields in the enclosure were quite different depending on the positions of the magnet. Thus, it is valuable to study the thermomagnetic convection inside the enclosure for different magnet positions with the poles of the magnet paralleling to bottom wall of the enclosure. 


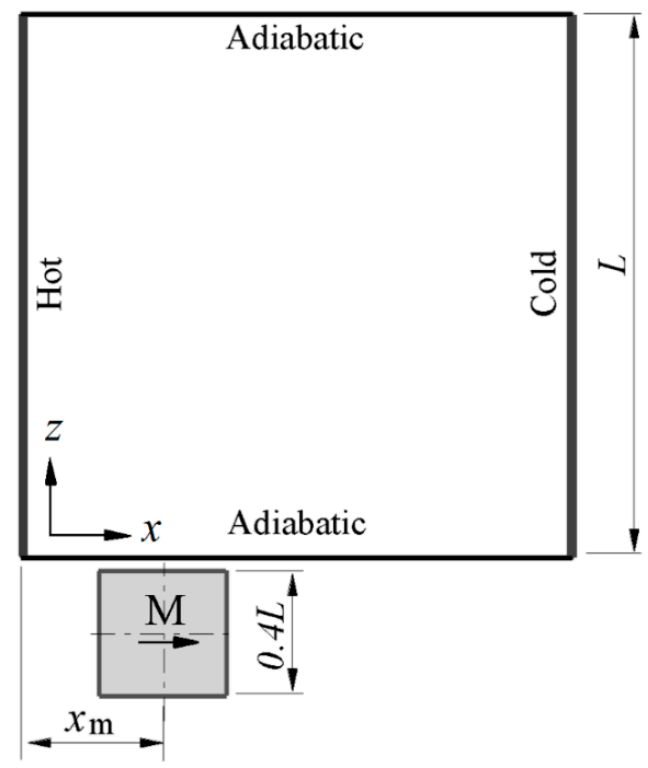

Figure 1. Schematic view of the physical model.

Table 1. Parameters and physical properties of oxygen [15] (300 K, $1 \mathrm{~atm})$.

\begin{tabular}{cccc}
\hline$B r$ & $0.5 \mathrm{~T}-3.0 \mathrm{~T}$ & $\mu$ & $20.65 \times 10^{-6} \mathrm{~Pa} \cdot \mathrm{s}$ \\
$x_{\mathrm{m}} / L$ & $0.0-1.0$ & $\lambda$ & $2.65 \times 10^{-2} \mathrm{~W} /(\mathrm{m} \cdot \mathrm{K})$ \\
$T_{\mathrm{h}}$ & $308.6 \mathrm{~K}$ & $\alpha$ & $22.136 \times 10^{-6} \mathrm{~m}^{2} / \mathrm{s}$ \\
$T_{\mathrm{c}}$ & $300 \mathrm{~K}$ & $\chi$ & $1.738 \times 10^{-6} \mathrm{~m}^{3} / \mathrm{kg}$ \\
$P r$ & 0.717 & $T$ & $300.0 \mathrm{~K}$ \\
$\rho$ & $1.301 \mathrm{~kg} / \mathrm{m}^{3}$ & $\beta$ & $1 / 300.0(1 / \mathrm{K})$ \\
$v$ & $15.878 \times 10^{-6} \mathrm{~m}^{2} / \mathrm{s}$ & $\mu_{\mathrm{m}}$ & $4 \pi \times 10^{-7} \mathrm{H} / \mathrm{m}$ \\
\hline
\end{tabular}

(a)

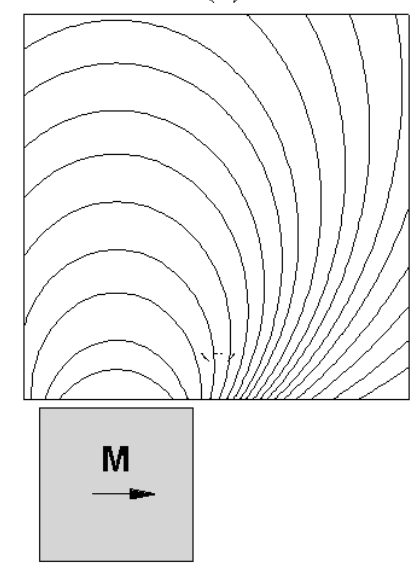

(b)

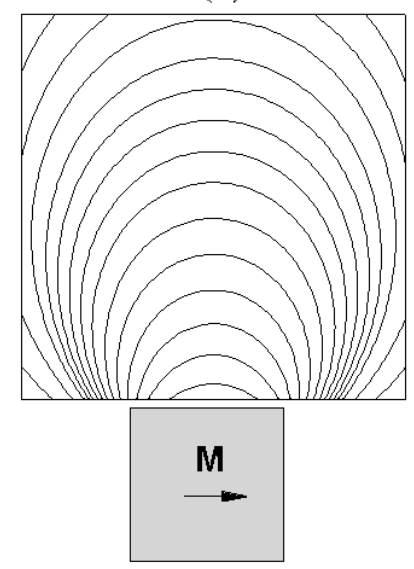

(c)

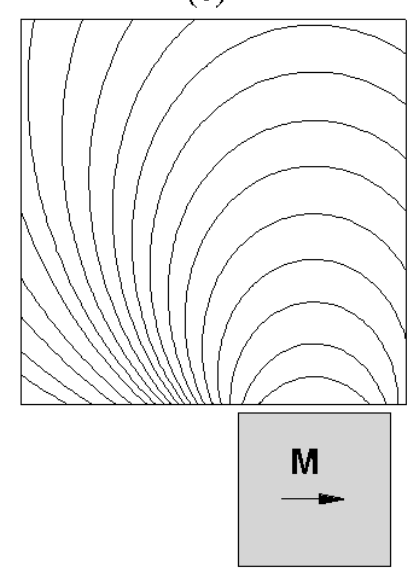

Figure 2. Magnetic field lines in the enclosure for different magnet positions. (a) $x_{\mathrm{m}} / L=0.2$, (b) $x_{\mathrm{m}} / L=0.5$, (c) $x_{\mathrm{m}} / L=0.8$.

\section{Mathematical Equations}

The magnetizing force, which is similar to the gravitational force, is a conservative force. Thus, both magnetic field gradient and the temperature gradient are necessary for thermomagnetic convection. The normal expression of magnetizing force is as follows $[15,18]$ :

$$
f_{\mathrm{m}}=\mu_{\mathrm{m}} \rho \chi \nabla \boldsymbol{H}^{2} / 2 \approx \rho \chi \nabla \boldsymbol{B}^{2} /\left(2 \mu_{\mathrm{m}}\right) .
$$


The magnetic induction $\boldsymbol{B}$ can be expressed as

$$
B=\nabla \times A .
$$

where $A$ is the vector potential generated by the permanent magnet at the calculated point. The calculation of magnetic induction $\boldsymbol{B}$ refers to Reference [15].

The momentum equation including the magnetizing force is as follows:

$$
\rho(\boldsymbol{u} \cdot \nabla) \boldsymbol{u}=-\nabla p+\mu \nabla^{2} \boldsymbol{u}+\rho \chi \nabla \boldsymbol{B}^{2} /\left(2 \mu_{\mathrm{m}}\right) .
$$

The gas is assumed as an incompressible Newtonian fluid, and the Boussinesq approximation that density is assumed to be constant except for the density difference for buoyancy force was employed. The parameters under the isothermal state are marked $\rho_{0}, p_{0}$, and $\chi_{0}$ at reference temperature $T_{0}$.

$$
0=-\nabla p_{0}+\rho_{0} \chi_{0} \nabla \boldsymbol{B}^{2} /\left(2 \mu_{\mathrm{m}}\right) .
$$

When there is a temperature difference, the magnetic susceptibility and density also change with temperature. Subtracting Equation (4) from Equation (3), we get

$$
\rho(\boldsymbol{u} \cdot \nabla) \boldsymbol{u}=-\nabla\left(p-p_{0}\right)+\mu \nabla^{2} \boldsymbol{u}+\left(\rho \chi-\rho_{0} \chi_{0}\right) \nabla \boldsymbol{B}^{2} /\left(2 \mu_{\mathrm{m}}\right) .
$$

The density can be expressed by a Taylor expansion around a reference state $T_{0}$ as follows:

$$
\rho \approx \rho_{0}+(\partial \rho / \partial T)_{0}\left(T-T_{0}\right) .
$$

On the other hand, the thermal volume coefficient of expansion $\beta$ for gas is

$$
\beta=-(\partial \rho / \partial T)_{\mathrm{p}} / \rho
$$

Then Equation (6) can be rewritten as follows by keeping only two terms of the Taylor expansion:

$$
\rho-\rho_{0}=(\partial \rho / \partial T)_{0}\left(T-T_{0}\right)=-\rho_{0} \beta_{0}\left(T-T_{0}\right) .
$$

With the ideal gas law, Equation (7) can be rewritten as

$$
\beta=-\frac{1}{\rho} \partial\left(\frac{p}{R_{g} T}\right) / \partial T=\frac{p}{\rho R_{g} T^{2}}=\frac{1}{T} .
$$

Using the Taylor expansion around a reference state $T_{0}$, the difference of $\rho \chi$ can be written as

$$
\rho \chi-(\rho \chi)_{0} \approx\left(\chi \frac{\partial \rho}{\partial T}+\rho \frac{\partial \chi}{\partial T}\right)_{0}\left(T-T_{0}\right) .
$$

The magnetic susceptibility of paramagnetic gas is a function of temperature according to the Curie law,

$$
\partial \chi / \partial T=\partial(C / T) / \partial T=-C / T^{2}=-\chi / T .
$$

Equation (10) can be rewritten as

$$
\left(-\rho \beta \chi-\frac{\rho \chi}{T}\right)_{0}\left(T-T_{0}\right)=-2 \rho_{0} \beta_{0} \chi_{0}\left(T-T_{0}\right) .
$$

Therefore, the momentum equation, Equation (5), can be written as follows:

$$
(\boldsymbol{u} \cdot \nabla) \boldsymbol{u}=-\nabla p / \rho_{0}+\mu / \rho_{0} \nabla^{2} \boldsymbol{u}-\beta_{0} \chi_{0}\left(T-T_{0}\right) / \mu_{\mathrm{m}} \nabla \boldsymbol{B}^{2} .
$$


The continuity equation is

$$
\nabla \cdot \boldsymbol{u}=0
$$

The energy equation is

$$
\rho c_{p}(\boldsymbol{u} \cdot \nabla) T=\lambda \nabla^{2} T .
$$

The temperature of the cold wall is selected as the reference temperature, $T_{0}=T_{\mathrm{c}}$. The initial conditions for the gas in the enclosure are $u=w=0$, and $T=T_{0}$, and no-slip conditions are applied on the walls of the enclosure.

The dimensionless parameters used are Nusselt numbers on the hot and cold walls. Local Nusselt number $N u_{\mathrm{h}}$ and $N u_{\mathrm{c}}$ on the hot and cold walls are as follows:

$$
\begin{aligned}
& N u_{\mathrm{h}}=\frac{L}{T_{\mathrm{h}}-T_{\mathrm{c}}}\left|\frac{\partial T}{\partial x}\right|_{x=0}, \\
& N u_{\mathrm{c}}=\frac{L}{T_{\mathrm{h}}-T_{\mathrm{c}}}\left|\frac{\partial T}{\partial x}\right|_{x=L} .
\end{aligned}
$$

The averaged Nusselt number is obtained by averaging $N u_{\mathrm{h}}$ on the hot wall,

$$
N u=\frac{1}{L} \int_{L} N u_{\mathrm{h}} d z \text {. }
$$

\section{Grid Dependence and Validation}

The grid dependence study was carried out between three grid systems $82 \times 82,102 \times 102$ and $122 \times 122$. The magnet was located at $x_{\mathrm{m}} / L=0.0$ with $\mathrm{Br}=2.0 \mathrm{~T}$. The fine grid was about 2.2 times larger than the coarse grid. The distributions of $N u_{\mathrm{h}}$ on the hot wall and $N u_{\mathrm{c}}$ on the cold wall for different grid systems are presented in Figure 3. As the magnetic field was non-uniform, the distribution of $N u_{\mathrm{h}}$ on the hot wall was quite different from $N u_{\mathrm{c}}$ on the cold wall, which was also quite different from the distributions of $N u_{\mathrm{h}}$ and $N u_{\mathrm{c}}$ under a gravity field, as reported in Reference [13]. The results of local $\mathrm{Nu}$ on both hot and cold walls are nearly the same for the three different grid systems studied. Thus, the results are grid independent in a large range of grid numbers and the medium-size grid system, $102 \times 102$, was selected for all computations in order to save computation resources.

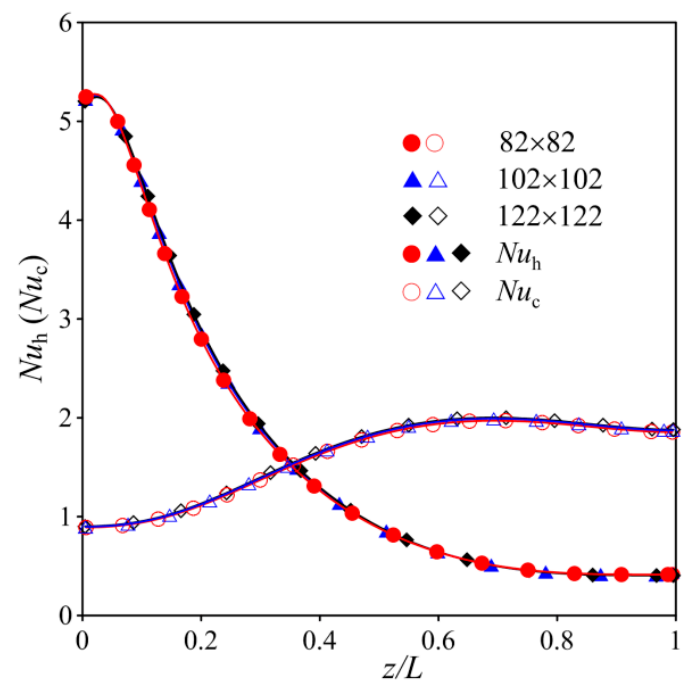

Figure 3. Grid dependence study.

In order to validate the numerical method, the natural convection in a square enclosure filled with air, reported by De Vahl Davis [19], was tested and compared with the reported results. The comparisons 
of the average values of $\mathrm{Nu}$ on the hot wall are shown in Table 2. The averaged Nusselt number agrees well with the results reported by De Vahl Davis [19]. The maximum difference was less than $0.05 \%$.

Table 2. Comparison of numerical results with previous work $\left(R a=10^{5}\right)$.

\begin{tabular}{cccc}
\hline Nusselt Number & De Vahl Davis [19] & Present & Maximum Error \\
\hline$N u$ & 4.519 & 4.521 & $0.05 \%$ \\
\hline
\end{tabular}

\section{Results and Discussion}

\subsection{Effect of Location of Magnet on Temperature}

The distributions of temperature in the enclosure with different magnet locations are shown in Figure 4 for $B r=2.0 \mathrm{~T}$. The magnet locations in Figure $4 \mathrm{a}-\mathrm{d}$ are $x_{m} / L=0.2,0.4 .0 .6$ and 1.0, respectively. As the magnetic field depends on the location of the magnet, the temperature fields for different magnet locations were quite different from each other. The temperature near the hot wall increased quickly from the bottom to the top of the enclosure and a large temperature gradient existed near the lower part of the hot wall. As the fluid washed toward the hot wall near the bottom wall, the temperature layer was thin near the lower part of the hot wall. The temperature near the upper part of the hot wall was high due to the strong convection near the lower part of the hot wall. Comparing Figure $4 \mathrm{~b}$ with Figure $4 a$, the temperature gradient near the lower part of the hot wall in Figure $5 b$ is slightly larger than that in Figure 4a. When the magnet location changed from $x_{m} / L=0.4$ to 1.0, the temperature gradient near the lower part of the hot wall decreased while the temperature gradient near the upper part of the hot wall increased, meaning the heat transfer on the lower part of the hot wall decreased and the heat transfer on the upper part of the hot wall increased. When the magnet was fixed at $x_{m} / L=1.0$, as shown in Figure $4 \mathrm{~d}$, the temperature near the hot wall increased gradually and the difference in temperature gradient near the hot wall was small compared with Figure 4a-c, meaning that the heat transfer near the hot wall was quite weak when the magnet was located at $x_{m} / L=1.0$.

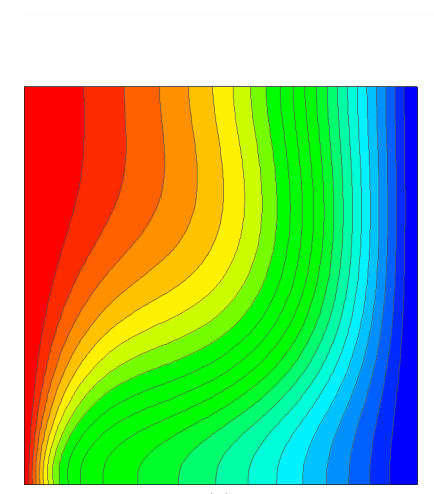

(a)

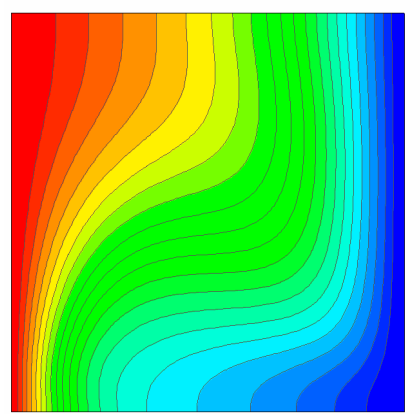

(c)

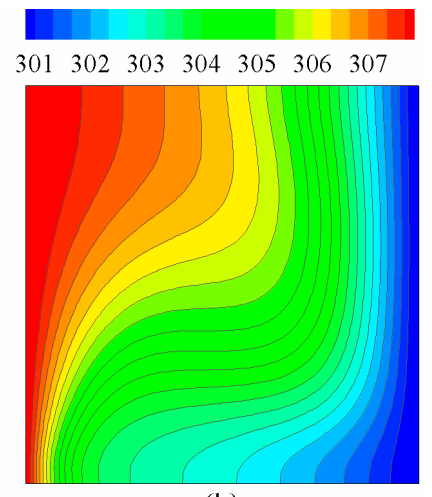

(b)

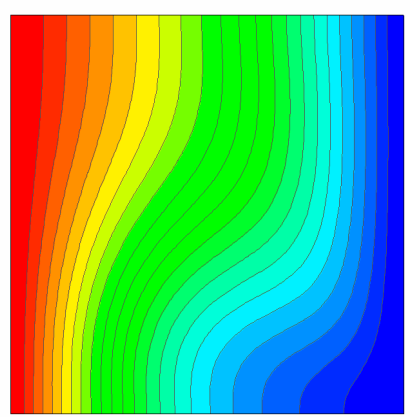

(d)

Figure 4. Temperature field for different locations of the magnet with $B r=2.0 \mathrm{~T}$; (a) $x_{m} / L=0.2$, (b) $x_{m} / L=0.4$, (c) $x_{m} / L=0.6$, and (d) $x_{m} / L=1.0$. 

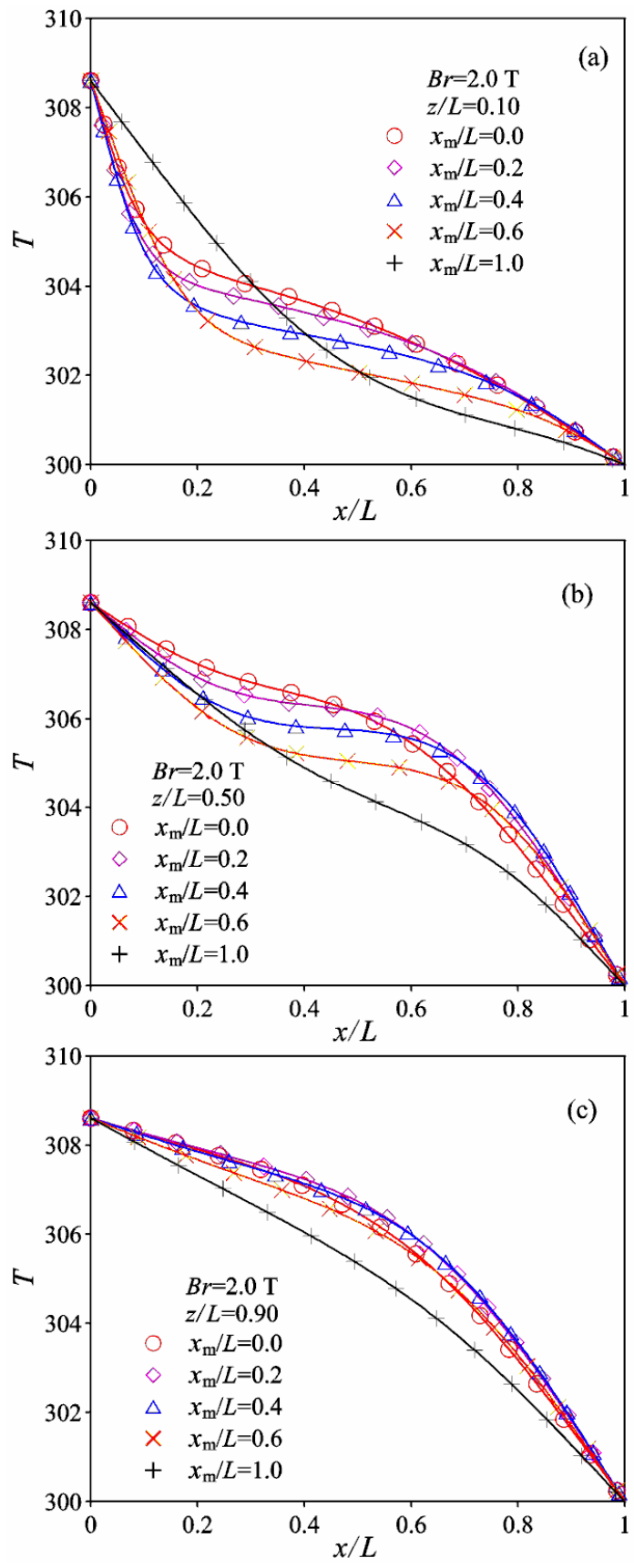

Figure 5. Distribution of $T$ on the selected lines; (a) $z / L=0.1,(\mathbf{b}) z / L=0.5$, and (c) $z / L=0.9$.

Figure 5 shows the distribution of temperature on the selected lines at $z / L=0.10,0.50$ and 0.90 for different magnet locations. The different locations of the magnet had an obvious effect on the distribution of temperature of the magnetic field. The temperature decreased from the hot wall to the cold wall. On the line at $z / L=0.10$ there was large temperature gradient near the hot wall. The temperature decreased quickly in the region with $x / L$, changing from 0.0 to around 0.2 . Then, the value of the temperature decreased gradually until the cold wall. The temperature gradient near the hot wall first increased a little when the location of magnet changed from $x_{m} / L=0.0$ to 0.4 , then the temperature gradient decreased when the location of magnet changed from $x_{m} / L=0.4$ to 1.0. Obvious differences in $N u$ exist on the line at $z / L=0.10$ in the range of $x / L$ between 0.2 and 0.8 . On the line at $z / L=0.50$, the temperature was higher than that of the line at $z / L=0.10$, and the temperature gradient was smaller than that of the line at $z / L=0.10$. Meanwhile, the temperature gradient near the cold wall was higher than that of the line at $z / L=0.10$. On the line at $z / L=0.90$, as shown in Figure $5 c$, 
the temperature gradient near the hot wall continued to decrease and the temperature decreased gradually from the hot wall to the cold wall. The temperature gradient near the cold wall was larger than that near the hot wall on the line at $z / L=0.90$.

\subsection{Effect of Location of Magnet on Flow Field}

Figure 6 shows the flow field for different locations of the magnet with $B r=2.0 \mathrm{~T}$. The magnet locations for Figure $6 \mathrm{a}-\mathrm{d}$ were $x_{m} / L=0.2,0.4,0.6$ and 1.0 , respectively. The velocity fields in the square enclosure were quite different from each other for different magnet locations. Large velocities were located in the region where the magnetic field was strong. There was one vortex rotating in a clockwise direction near the bottom wall of the enclosure and the vortex moved along with the moving of the magnet locations. The center of the vortex moved from the left to the right of the enclosure when the magnet location moved from left to right. In Figure 6a, the gas with a large velocity flowed along the bottom wall and washed toward the lower part of the hot wall under the driving magnetic force. The vortex increased a little when the magnet location moved from $x_{m} / L=0.2$ to $x_{m} / L=0.4$, as shown in Figure 6a,b. The vortex moved away from the bottom left corner, and the zone with a large velocity also increased compared with that in Figure 6a. The strength of the vortex also decreased and the center of the vortex kept moving away from the left wall and toward the right wall, along with the magnet location as it changed from $x_{m} / L=0.4$ to $x_{m} / L=1.0$. When the magnet was placed at $x_{m} / L=1.0$ in Figure $6 \mathrm{~d}$, the flow field became very weak. Thus, the flow field was obviously affected by the magnet location.

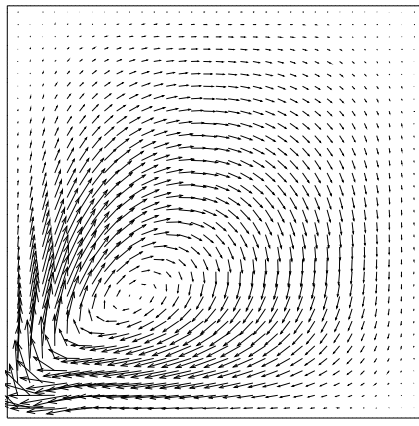

(a)

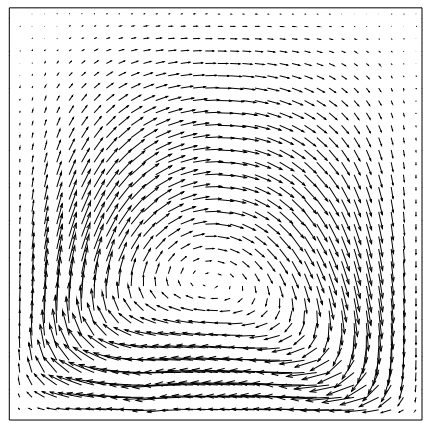

(c)

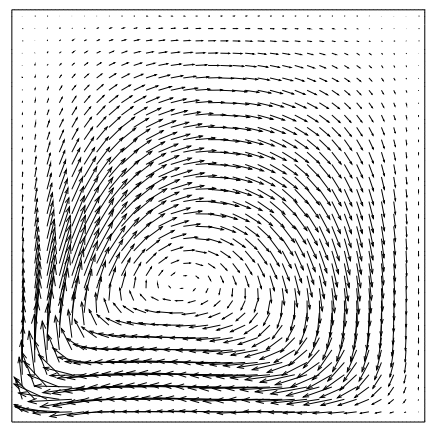

(b)

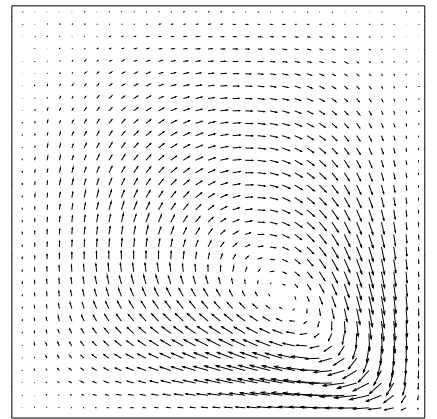

(d)

Figure 6. Flow fields for different locations of the magnet with $B r=2.0 \mathrm{~T} ;(\mathbf{a}) x_{m} / L=0.2,(\mathbf{b}) x_{m} / L=0.4$, (c) $x_{m} / L=0.6$, and (d) $x_{m} / L=1.0$.

\subsection{Effect of Location of Magnet on Buoyancy Force}

Figure 7 shows the vector plot of $\boldsymbol{F}$ inside the enclosure for different locations of the magnet. The combined contour plot relates to the buoyancy force $F_{z}$. As the magnetic field decreased quickly in the short distance from the magnet surface, there was a large value $\boldsymbol{F}$ only in a small region around the magnet. As the magnetic buoyancy force related to the combination of temperature and magnetic field, the values of $F$ were not symmetric about the z-center-line of the magnet, although the magnetic 
field was symmetric about the $\mathrm{z}$-center-line of the magnet. The temperature decreased from the hot wall to the cold wall, thus $\boldsymbol{F}$ decreased gradually, along with the magnet moving from the hot wall to the cold wall. The values of $\boldsymbol{F}$ near the left corner of the magnet were obviously larger than that near the right corner of the magnet. The value $F_{Z}$ was positive in the enclosure because the magnet was located below the enclosure. The value $F_{\mathrm{x}}$ was a little complicated. $F_{\mathrm{x}}$ was positive on the right of the magnet and negative on the left of the magnet. $F_{\mathrm{X}}$ was positive between the left pole and $z$-center-line of the magnet, while it was negative between the $z$-center-line and the right pole of the magnet.

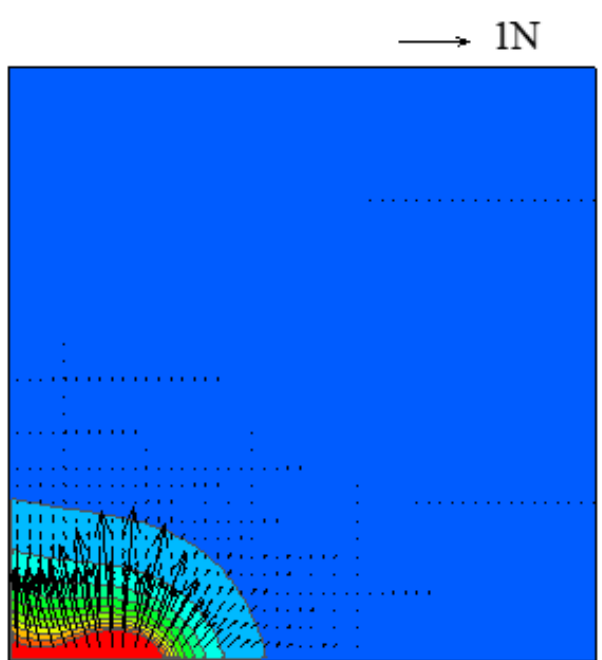

(a)

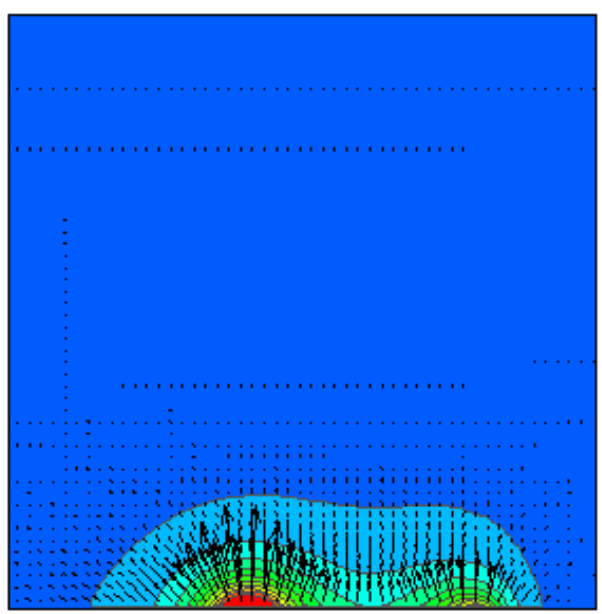

(c)

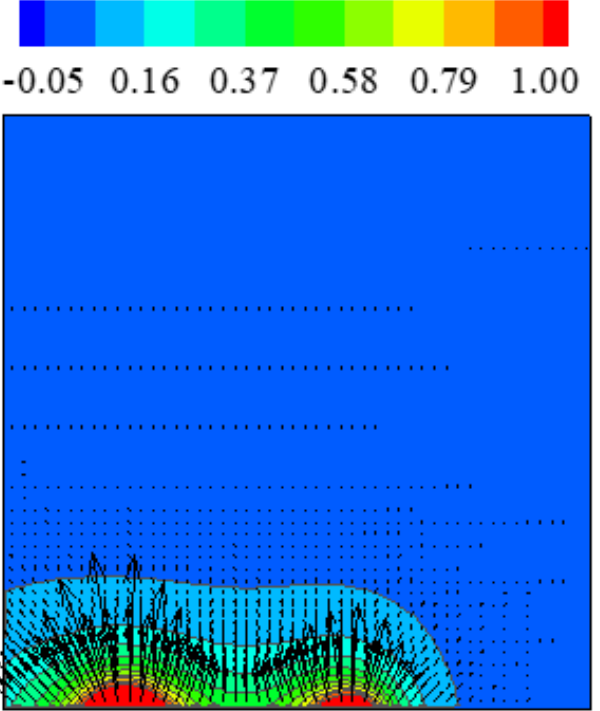

(b)

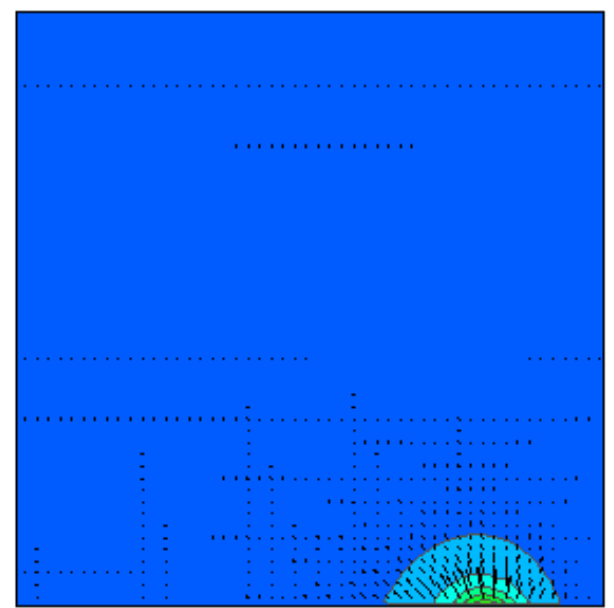

(d)

Figure 7. Distribution of $\boldsymbol{F}$ for different locations of the magnet with $B r=2.0 \mathrm{~T}$; (a) $x_{m} / L=0.2$, (b) $x_{m} / L=0.4$, (c) $x_{m} / L=0.6$, and (d) $x_{m} / L=1.0$.

Figure 8 shows the distribution of buoyancy force $F_{\mathrm{Z}}$ on the selected lines with different magnet locations for $\mathrm{Br}=2.0 \mathrm{~T}$. The magnetic buoyancy force relates not only to the temperature difference but also tightly to the gradient of the square of magnetic field strength. As there was a large gradient of the square of magnetic field strength near the corners of the magnet, large values of magnetic force $F_{z}$ were obtained near the corners of the magnet. The distribution line of $F_{z}$ was wave-like and there are two peak points according to the corners of the magnet. As the magnetic field was symmetric about the $z$-center-line of the magnet and the temperature generally decreased from the left wall to the right wall, the left peak point of $F_{\mathrm{Z}}$ was larger than the right peak point of $F_{\mathrm{z}}$. On the line at $z / L=0.10$, as shown 
in Figure 8a, the values of $F_{\mathrm{z}}$ near the hot wall increased first when the magnet location changed from $x_{\mathrm{m}} / L=0.0$ to $x_{\mathrm{m}} / L=0.2$. Then, the value of $F_{\mathrm{z}}$ near the hot wall decreased generally along with the moving of the magnet from $x_{\mathrm{m}} / L=0.2$ to $x_{\mathrm{m}} / L=1.0$. When the left pole of the magnet overlapped with the left wall, that is $x_{\mathrm{m}} / L=0.2$, the largest value of $F_{\mathrm{z}}$ was obtained near the hot wall because this was the location of the strongest magnetic field near the hot wall, where the temperature was also the highest. Then, the value of $F_{z}$ decreased quickly along $x$-direction due to the decrease in both temperature and magnetic field strength. Around the right corner of the magnet, where the magnetic field strength was high, the peak value of $F_{\mathrm{Z}}$ was also obtained, although the temperature was low.
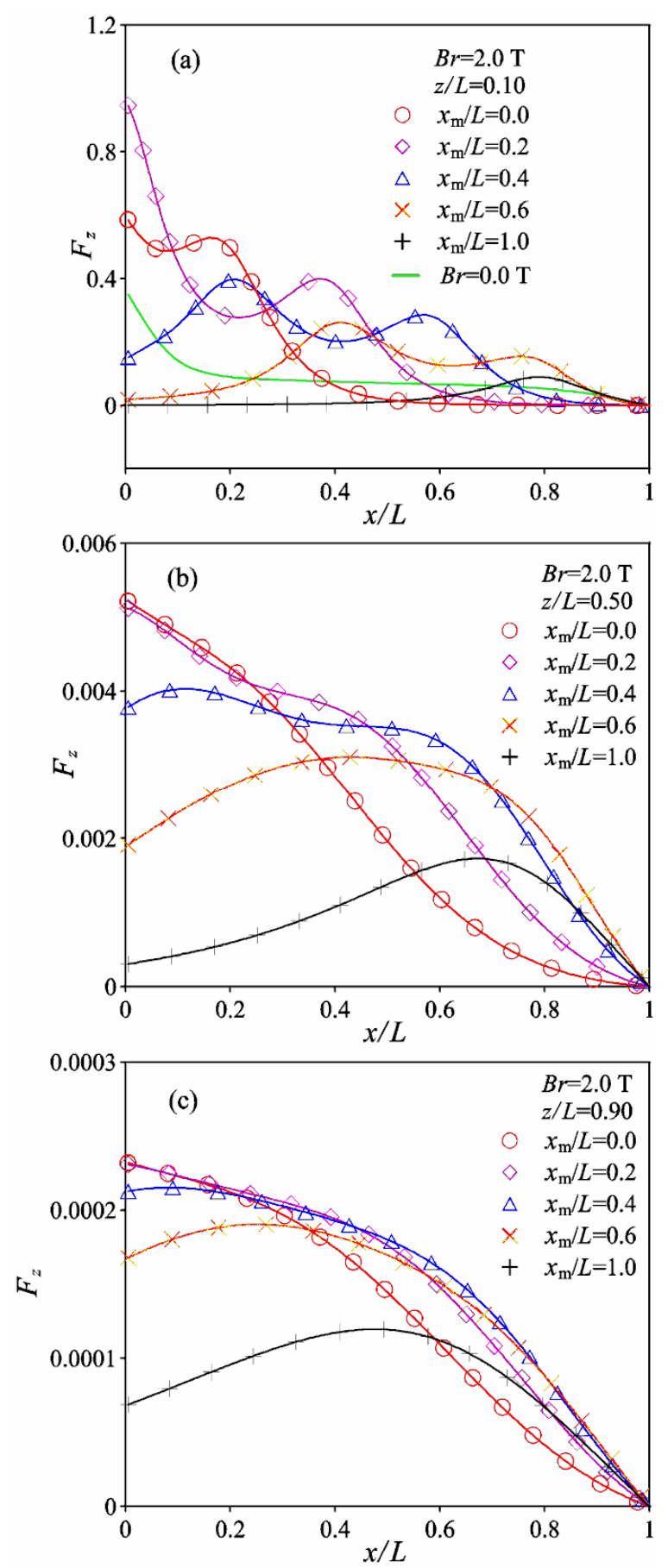

Figure 8. Effects of location of magnet on magnetic buoyancy force $F_{z},(\mathbf{a}) z / L=0.10,(\mathbf{b}) z / L=0.50$, and (c) $z / L=0.90$. 
When the magnet continued moving away from the hot wall, such as at $x_{\mathrm{m}} / L=0.4$, the magnetic field became weak near the hot wall and the value of $F_{z}$ near the hot wall decreased. The position of the peak value of $F_{z}$ also moved away from the hot wall and the value of $F_{z}$ decreased due to the decrease in temperature away from the hot wall. Meanwhile the difference in $F_{z}$ between the two peak values also decreased due to the decrease in the temperature gradient toward the cold wall, as shown in Figure 4 . When the magnet was located at $x_{\mathrm{m}} / L=1.0$, with the cold wall overlapping with the center of the magnet, the magnetic field around the hot wall was very weak and the value of $F_{\mathrm{Z}}$ was very small, close to zero. There was only one peak value of $F_{z}$ near the cold wall corresponding to the strong magnetic field near the left corner of the magnet. On the lines at $z / L=0.50$ and 0.90 , as shown in Figure $8 b, c$, the values of $F_{z}$ were smaller, compared with those shown in Figure 8a, due to the weak magnetic field in the region far away from the bottom of the enclosure. As the magnetic field was weak and the difference in magnetic field strength on the selected lines was quite small, there was a slight effect of corners of the magnet on the values of $F_{\mathrm{z}}$. Thus, the peak values of $F_{\mathrm{Z}}$ related to the corners of the magnet were not obvious at $z / L=0.50$ and 0.90 . The magnetic buoyancy force $F_{z}$ was mainly dominated by the temperature on the upper part of the enclosure where the magnetic field was quite weak.

The distributions of $F_{x}$ on the selected lines located at $z / L=0.10,0.50$ and 0.90 are presented in Figure 9 for different magnet positions. $F_{x}$ was zero at the point of the intersection of the selected line and the $z$-center-line of the magnet because of the zero value of the magnetic field gradient along the $x$-direction at the intersection point. On the line at $z / L=0.1$, as shown in Figure 9 a, the value of $F_{x}$ was nearly zero near the hot wall when the magnet was located at $x_{\mathrm{m}} / L=0.0$. The value of $F_{x}$ was negative in the region between $x / L=0.0$ and 0.20 , and $F_{x}$ was zero at the points ahead of $x / L=0.2$, corresponding to the right corner of the magnet. Then, $F_{x}$ became positive and reached maximum value in the region behind the corner of the magnet. The value of $F_{x}$ first decreased quickly a short distance away from the pole of the magnet and then decreased gradually until zero on the cold wall. The value of $F_{x}$ was also nearly zero around the position at $x / L=0.4$ when the magnet was located at $x_{\mathrm{m}} / L=0.4$. In the region between $x / L=0.4$ and 1.0 , the thermomagnetic convection was affected by the right part of the magnetic field and the distribution of $F_{x}$ was similar to that of the magnet location at $x_{\mathrm{m}} / L=0.4$. In the region between $x / L=0.0$ and 0.4 , the thermomagnetic convection was affected by the left part of the magnetic field. The value $F_{x}$ was negative in the region between $x / L=0.0$ and 0.20 , which was on the left of the left pole of the magnet. In the region between $x / L=0.2$ and $0.4, F_{x}$ was positive and the peak value of $F_{x}$ was obtained between $x / L=0.2$ and 0.4 . When the magnet moved from $x_{\mathrm{m}} / L=0.0$ to $x_{\mathrm{m}} / L=1.0$, the peak value of $F_{x}$, due to the right pole of the magnet and the peak value in accordance with the region between the poles, decreased gradually due to the decrease in temperature. The peak value of $F_{x}$, due to the left pole of the magnet, increased first when the magnet moved from $x_{\mathrm{m}} / L=0.2$ to $x_{\mathrm{m}} / L=0.4$ and then decreased gradually from $x_{\mathrm{m}} / L=0.4$ to $x_{\mathrm{m}} / L=1.0$. There was no peak point related to the left pole of the magnet for the location of the magnet at $x_{\mathrm{m}} / L=0.0$, because the $z$-center-line of the magnet overlapped with the hot wall and the thermomagnetic convection in the enclosure was mainly affected by the right part of the magnetic field. On the lines at $z / L=0.5$ and 0.9 , as shown in Figure $9 b, c$, the magnetic fields were quite weak and $F_{x}$ was also quite small compared with that of the line at $z / L=0.1$. The value of $F_{x}$ was positive in the region covered by the right part of the magnetic field, and a negative value of $F_{x}$ was obtained in the region covered by the left part of the magnetic field. 

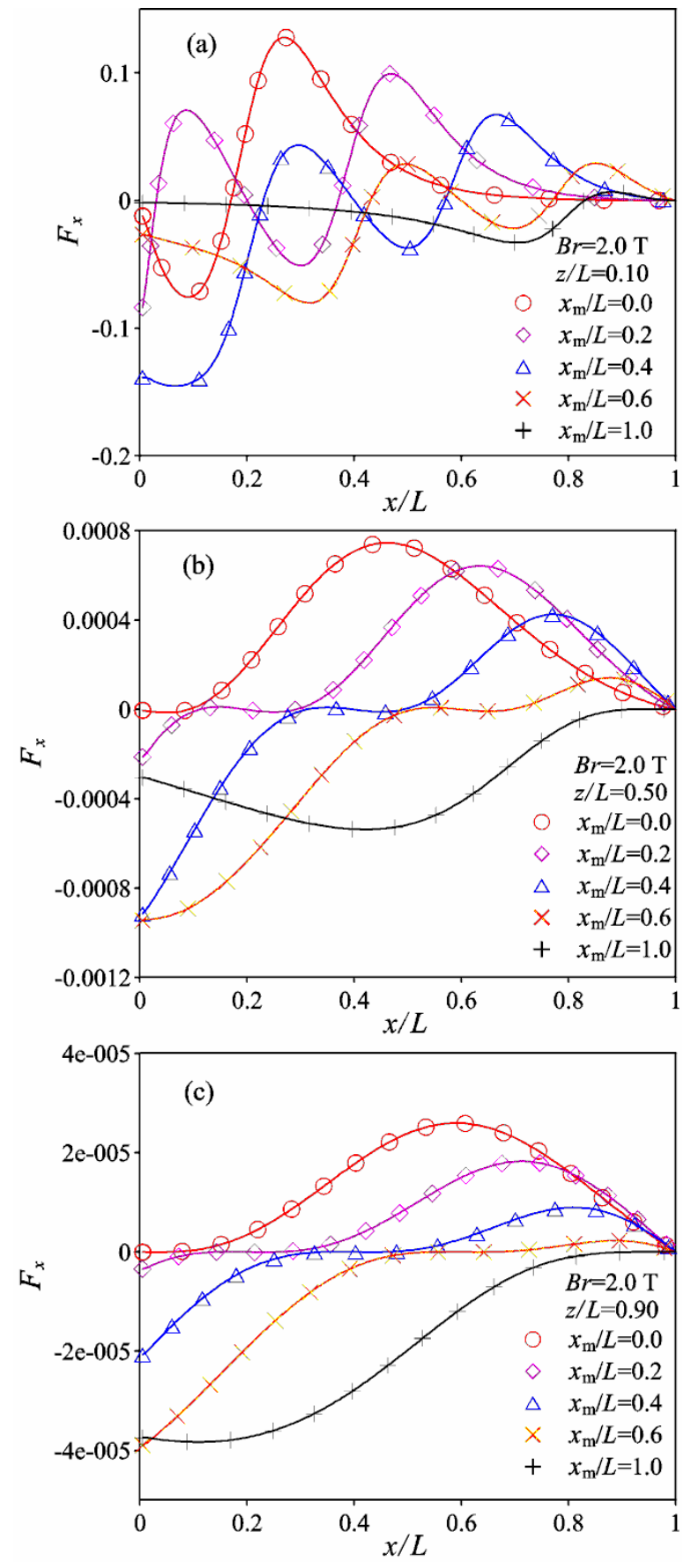

Figure 9. Distribution of $F_{x}$ on the selected lines; (a) $z / L=0.1,(\mathbf{b}) z / L=0.5$, and (c) $z / L=0.9$.

\subsection{Effect of Location of Magnet on $\mathrm{Nu}$}

Figure 10 shows the effect of the location of the magnet with $B r=2.0 \mathrm{~T}$ on the distribution of $N u_{\mathrm{h}}$ on the hot wall and $N u_{\mathrm{c}}$ on the cold wall. The maximum value of $N u_{\mathrm{h}}$ was obtained near the bottom wall due to the combination of the high strength of magnetic field and temperature. The maximum value of $N u_{\mathrm{h}}$ first increased, with the magnet moving from $x_{\mathrm{m}} / L=0.0$ to $x_{\mathrm{m}} / L=0.3$, and got the largest maximum value when the magnet was located at $x_{\mathrm{m}} / L=0.3$. Then, the maximum value of $N u_{\mathrm{h}}$ for different magnet locations decreased when the magnet moved from $x_{\mathrm{m}} / L=0.3$ to $x_{\mathrm{m}} / L=1.0$. The maximum value for the case with $x_{\mathrm{m}} / L=0.3$ was about 3.6 times larger than that of the case with $x_{\mathrm{m}} / L=1.0$, and about $43 \%$ larger than when the magnet was located at the center with $x_{\mathrm{m}} / L=0.5$. $N u_{\mathrm{h}}$ decreased along the hot wall due to the increase in temperature and decrease in temperature 
gradient when the fluid, with high temperature flowing upward the hot wall. $N u_{\mathrm{h}}$ on the upper part of the hot wall generally increased with the increase of the value of $x_{\mathrm{m}} / L$. In addition, the difference in $N u_{\mathrm{h}}$ between cases with different magnet locations was much smaller compared with that on the lower part of the hot wall. The maximum values of $N u_{\mathrm{c}}$ on the cold wall were much smaller than $N u_{\mathrm{h}}$ on the hot wall. The values of $N u_{\mathrm{c}}$ on the upper part of the cold wall were higher than that on the lower part of the cold wall, which was converse to the distribution of $N u_{\mathrm{h}}$ on the hot wall. In the regions of the upper part of the hot wall and the lower part of the cold wall, the values of both $N u_{\mathrm{h}}$ and $N u_{\mathrm{c}}$ were less than 1. The reason is that the temperature gradients, as shown in Figure 5, are quite small in the regions near the upper part of the hot wall and the lower part of the cold wall. Thus, the conduction in the regions with a small temperature gradient was suppressed due to the thermomagnetic convection and the values of $N u_{\mathrm{h}}$ and $N u_{\mathrm{c}}$ were less than 1.
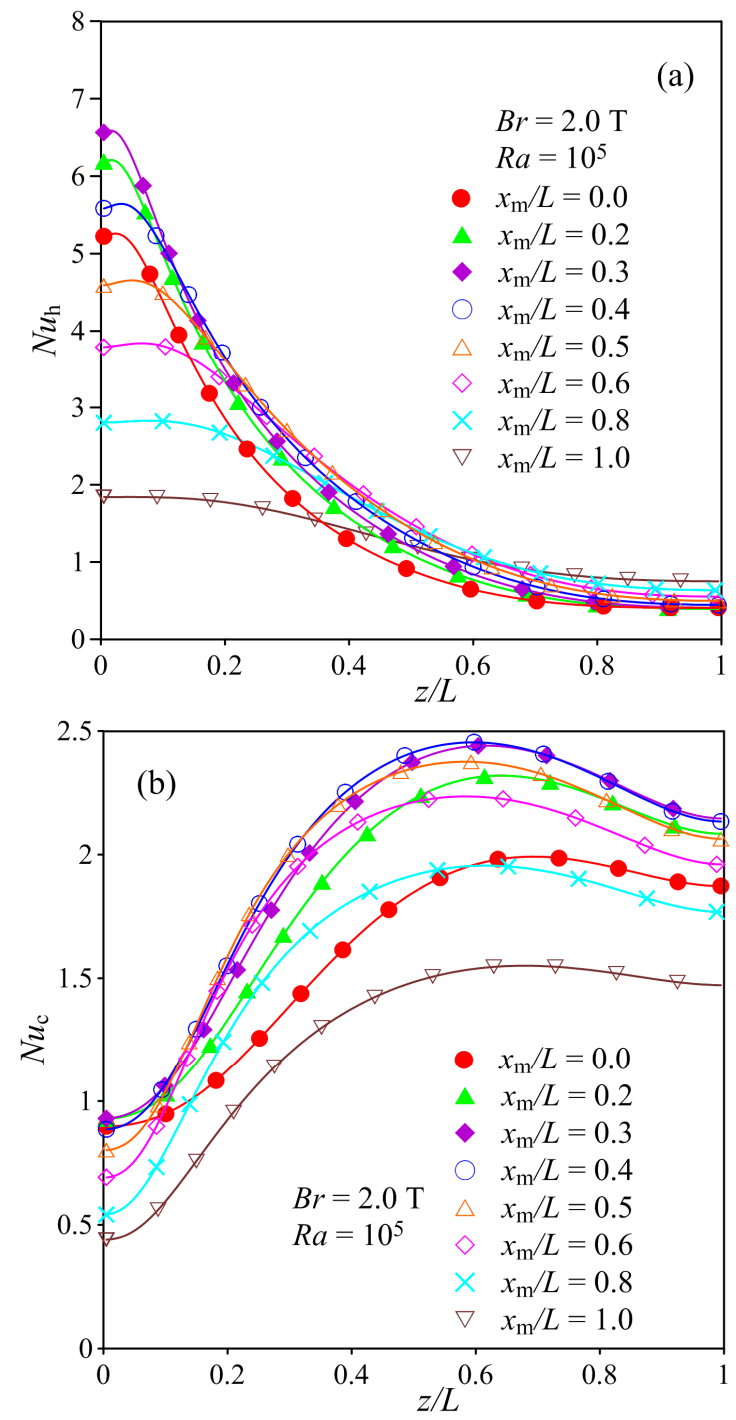

Figure 10. Effect of location of magnet on local $N u$; (a) $N u_{\mathrm{h}}$ and (b) $N u_{\mathrm{c}}$.

Figure 11 shows the effect of magnet strength on the distributions of $N u_{\mathrm{h}}$ and $N u_{\mathrm{c}}$ on the hot and cold walls when the magnet was located at $x_{\mathrm{m}} / L=0.4$. In the lower part of the hot wall, $N u_{\mathrm{h}}$ increased with the increase of $B r$. While in the upper part of the hot wall with $z / L>0.6$, the difference in the value of $N u_{\mathrm{h}}$ was slight and the values were nearly the same for $\mathrm{Br}>1.0 \mathrm{~T}$. The difference in $N u_{\mathrm{h}}$ on the lower part of the hot wall decreased with the increase of $\mathrm{Br}$. The maximum value of $N u_{\mathrm{h}}$ for $\mathrm{Br}=3.0 \mathrm{~T}$ was about 5.24 times larger than that for $\mathrm{Br}=0.5 \mathrm{~T}$. Nu $u_{\mathrm{c}}$ increased first away from the 
bottom and reached its maximum value around the center of the cold wall. Then, $N u_{\mathrm{c}}$ decreased from the maximum value point until the top. The value of $N u_{\mathrm{c}}$ near the top wall was higher than that near the bottom wall. Magnet strength had an obvious effect on the distribution of $N u_{\mathrm{c}}$, and $N u_{\mathrm{c}}$ generally increased with the increase of $B r$. In addition, the point with the maximum value of $N u_{\mathrm{c}}$ moved downward with increasing $B r$. The values of $N u_{\mathrm{h}}$ and $N u_{\mathrm{c}}$ were also less than 1 for some cases with different values of $\mathrm{Br}$ in the regions near the upper part of the hot wall and lower part of the cold wall. The reason was the same as that discussed in the figure, that the conduction in these regions was suppressed compared to the pure conduction in the enclosure with magnetic field.
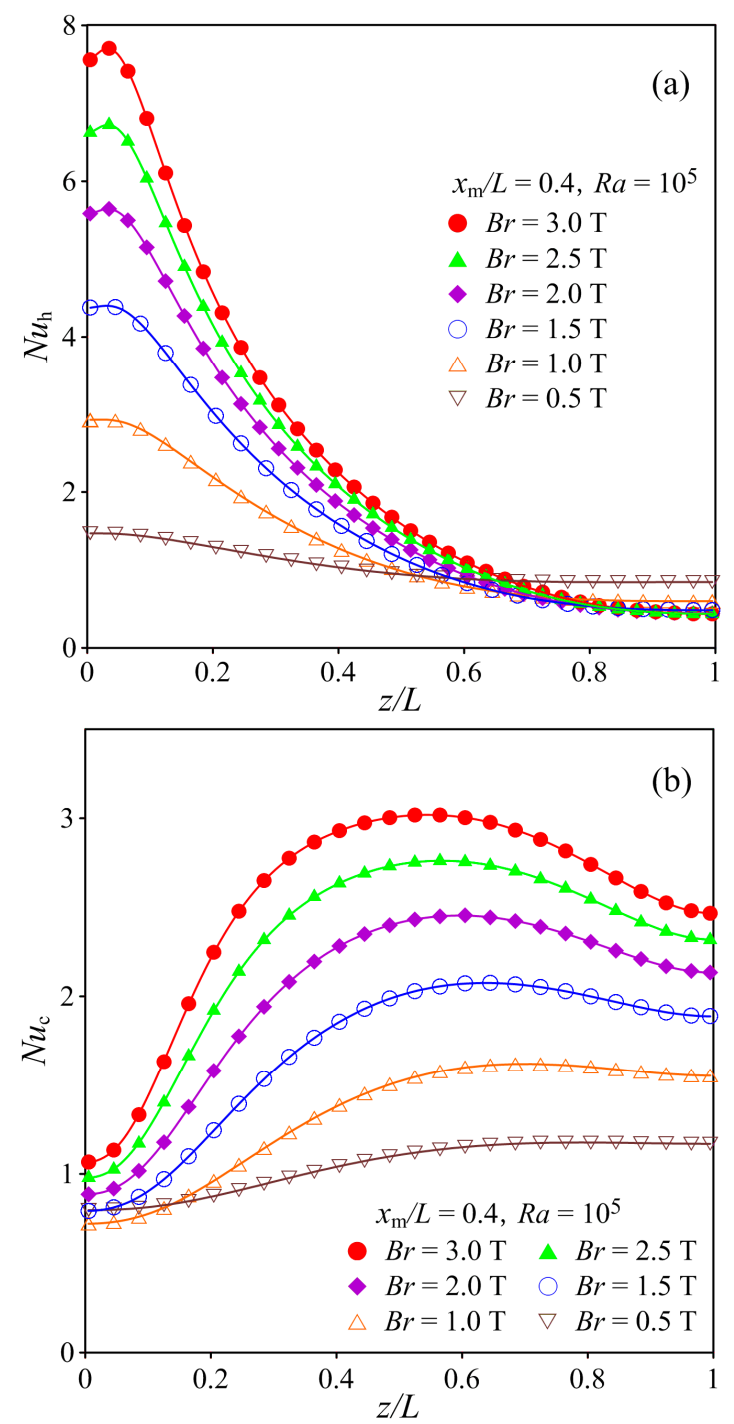

Figure 11. Effect of $B r$ on local $N u$ on the walls. (a) $N u_{\mathrm{h}}$, (b) $N u_{\mathrm{c}}$.

Figure 12 shows the distribution of averaged $N u$ in the enclosure as a function of the magnet location $x_{\mathrm{m}} / L$ and the different residual magnetic flux densities of $B r . N u$ increased with the increase of residual magnetic flux density. Under the same residual magnetic flux density, $\mathrm{Nu}$ first increased and then decreased in the studied range of magnet position between $x_{\mathrm{m}} / L=0.0$ and1.0. There was an optimum location of the magnet in terms of the largest value of $N u$ and the optimum locations of the magnet are slightly different from cases with different values of $B r$. The optimum location of the magnet for the cases with $B r=0.5,1.0,1.5,2.0,2.5$ and 3.0 T were $x_{\mathrm{m}} / L=0.5,0.4,0.4,0.4,0.36$ and 0.36 , respectively. The optimum location moved slightly toward the hot wall with the increase of $B r$. The maximum values of $\mathrm{Nu}$ at the optimum locations reached up to 2.54, 2.02 and 1.33 for $\mathrm{Br}=3.0 \mathrm{~T}$, 
2.0 T and $1.0 \mathrm{~T}$, respectively. Compared with the case of $x_{\mathrm{m}} / L=1.0$, the value of $N u$ at the optimum location increased by $77.0 \%, 69.4 \%, 60.0 \%, 46.1 \%, 25.2$ and $4.0 \%$ for different cases with $B r=3.0 \mathrm{~T}, 2.5 \mathrm{~T}$, $2.0 \mathrm{~T}, 1.5 \mathrm{~T}, 1.0 \mathrm{~T}$ and $0.5 \mathrm{~T}$, respectively. Thus, thermomagnetic convection can be further enhanced by optimizing magnet location.

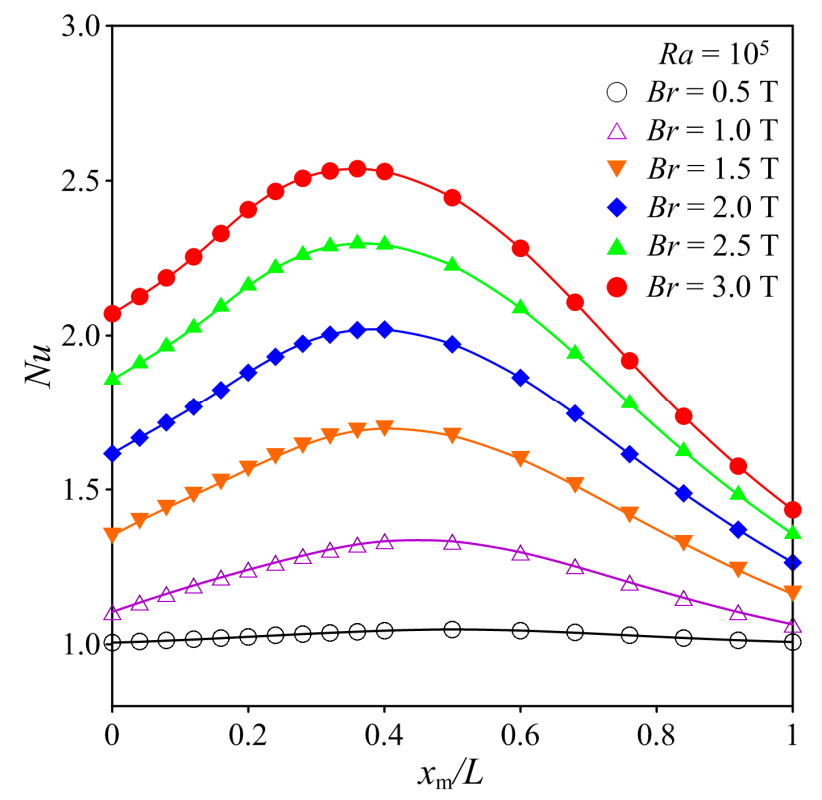

Figure 12. Effect of location of magnet on $\mathrm{Nu}$ for different values of $\mathrm{Br}$.

\section{Conclusions}

Thermomagnetic convection in an enclosure under a non-uniform magnetic field supplied by a permanent magnet at different locations was studied numerically. The permanent magnet was arranged in a novel manner, with the magnet being placed near the adiabatic wall with the poles parallel to the adiabatic wall. The main conclusions can be summarized as follows:

1. The location of magnet had a significant effect on the distribution of $N u$ on the hot wall, especially on the lower part of the hot wall. The maximum local $N u$ on the hot wall reached up to $2.9,5.6$ and 7.7 for $B r=1.0 \mathrm{~T}, 2.0 \mathrm{~T}$ and $3.0 \mathrm{~T}$, respectively.

2. The value of $\mathrm{Nu}$ on the upper part of the hot wall and on the lower part of the cold wall was less than 1.0, due to the suppression of conduction with a much lower temperature gradient caused by the thermomagnetic convection.

3. The optimum location of the magnet exists in terms of the largest value of $\mathrm{Nu}$. The optimum location of the magnet ranged from $x_{\mathrm{m}} / L=0.5$ to 0.36 , when the magnetic flux density increased from $0.5 \mathrm{~T}$ to $3.0 \mathrm{~T}$. Compared with the case of $x_{\mathrm{m}} / L=1.0$, the average value of $N u$ in the enclosure could be increased by up to $77 \%$ by optimizing the location of the magnet for the studied range of parameters.

Author Contributions: Conceptualization, K.S. and T.T.; Methodology, K.S.; Data Curation, S.W., W.S. and S.Z.; Writing-Original Draft Preparation, K.S.; Writing-Review \& Editing, T.T.

Funding: This research was funded by the National Natural Science Foundation of China (51866007), the Gansu Provincial Natural Science Foundation (17JR5RA092) and Collaborative Innovation Team Project (2018C-13), and the Foundation of a hundred youth talents training program of Lanzhou Jiaotong University.

Conflicts of Interest: The authors declare no conflict of interest. 


\section{Nomenclature}

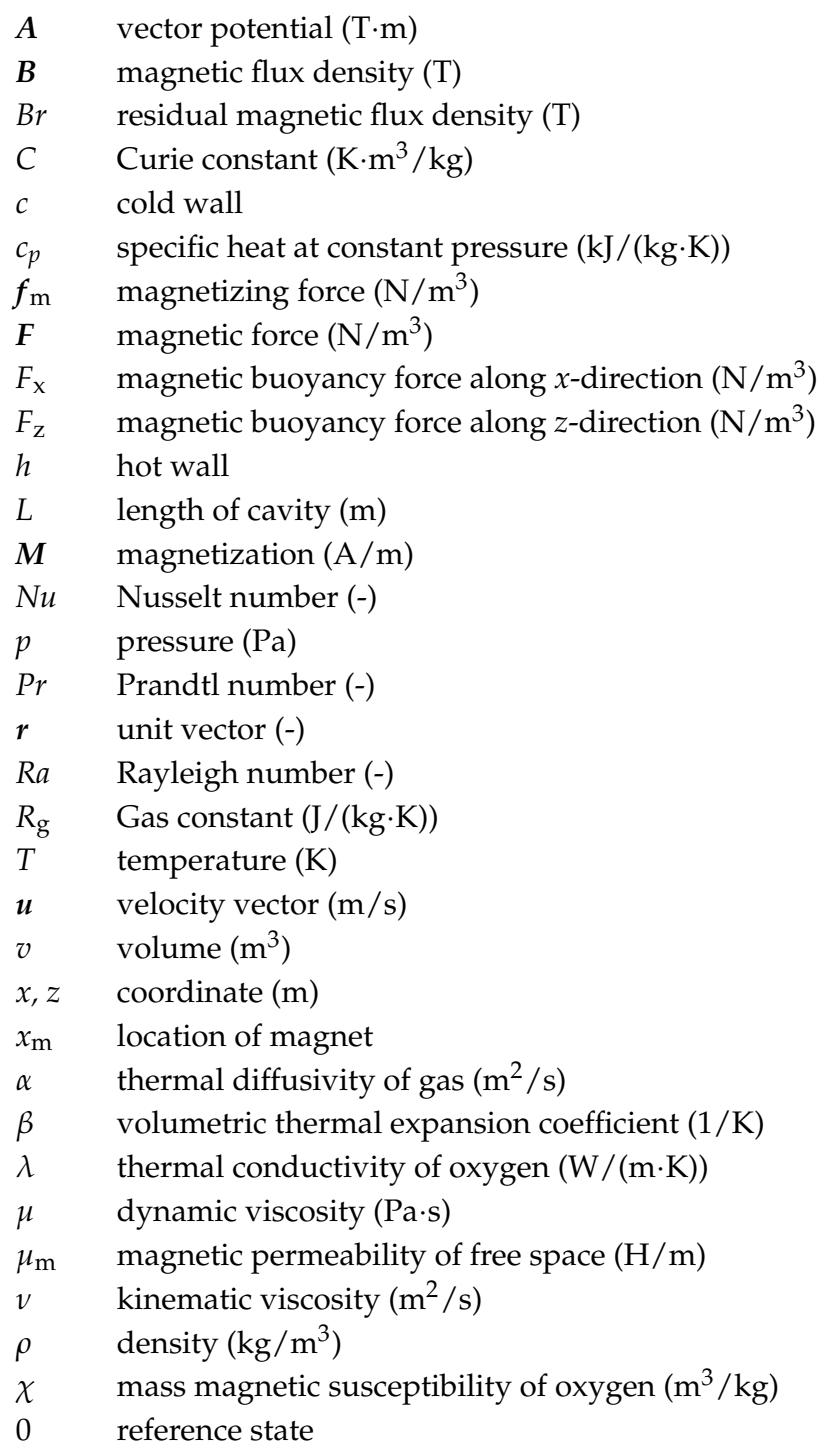

\section{References}

1. Das, D.; Roy, M.; Basak, T. Studies on natural convection within enclosures of various (non-square) shapes-A review. Int. J. Heat Mass Transf. 2017, 106, 356-406. [CrossRef]

2. Joudi, K.A.; Hussein, I.A.; Farhan, A.A. Computational model for a prism shaped storage solar collector with a right triangular cross section. Energy Convers. Manag. 2004, 45, 391-409. [CrossRef]

3. Kalaiselvam, S.; Veerappan, M.; Aaron, A.A.; Iniyan, S. Experimental and analytical investigation of solidification and melting characteristics of PCMs inside cylindrical encapsulation. Int. J. Therm. Sci. 2008, 47, 858-874. [CrossRef]

4. Mukhopadhyay, A.; Ganguly, R.; Sen, S.; Puri, I.K. A scaling analysis to characterize thermomagnetic convection. Int. J. Heat Mass Transf. 2005, 48, 3485-3492. [CrossRef]

5. Bahiraei, M.; Hangi, M. Flow and heat transfer characteristics of magnetic nanofluids: A Review. J. Magn. Magn. Mater. 2015, 374, 125-138. [CrossRef]

6. Krakov, M.S.; Nikiforov, I.V. To the influence of uniform magnetic field on thermomagnetic convection in square cavity. J. Magn. Magn. Mater. 2002, 252, 209-211. [CrossRef]

7. Krakov, M.S.; Nikiforov, I.V. Thermomagnetic convection in a porous enclosure in the presence of outer uniform magnetic field. J. Magn. Magn. Mater. 2005, 289, 278-280. [CrossRef] 
8. Tangthieng, C.; Finlayson, B.A.; Maulbetsch, J.; Cader, T. Heat transfer enhancement in ferrofluids subjected to steady magnetic fields. J. Magn. Magn. Mater. 1999, 201, 252-255. [CrossRef]

9. Yu, P.X.; Qiu, J.X.; Qin, Q.; Tian, Z.F. Numerical investigation of natural convection in a rectangular cavity under different directions of uniform magnetic field. Int. J. Heat Mass Transf. 2013, 67, 1131-1144. [CrossRef]

10. Tagawa, T.; Shigemitsu, R.; Ozoe, H. Magnetizing force modeled and numerically solved for natural convection of air in a cubic enclosure: Effect of the direction of the magnetic field. Int. J. Heat Mass Transf. 2002, 45, 267-277. [CrossRef]

11. Vatani, A.; Woodfield, P.L.; Nguyen, N.T.; Dao, D.V. Thermomagnetic convection around a current-carrying wire in ferrofluid. J. Heat Transf. 2017, 139, 104502. [CrossRef]

12. Vatani, A.; Woodfield, P.L.; Nguyen, N.T.; Dao, D.V. Onset of thermomagnetic convection around a vertically oriented hot-wire in ferrofluid. J. Magn. Magn. Mater. 2018, 456, 300-306. [CrossRef]

13. Jiang, C.W.; Shi, E.; Hu, Z.M.; Zhu, X.F.; Xie, N. Numerical simulation of thermomagnetic convection of air in a porous square enclosure under a magnetic quadrupole field using LTNE models. Int. J. Heat Mass Transf. 2015, 91, 98-109. [CrossRef]

14. Yang, L.J.; Ren, J.X.; Song, Y.Z.; Guo, Z.Y. Free convection of a gas induced by a magnetic quadrupole field. J. Magn. Magn. Mater. 2003, 261,377-384. [CrossRef]

15. Song, K.W.; Tagawa, T. Thermomagnetic convection of oxygen in a square enclosure under non-uniform magnetic field. Int. J. Therm. Sci. 2018, 125, 52-65. [CrossRef]

16. Ashouri, M.; Ebrahimi, B.; Shafii, M.B.; Saidi, M.H.; Saidi, M.S. Correlation for Nusselt number in pure magnetic convection ferrofluid flow in a square cavity by a numerical investigation. J. Magn. Magn. Mater. 2010, 322, 3607-3613. [CrossRef]

17. Szabo, P.S.B.; Früh, W.G. The transition from natural convection to thermomagnetic convection of a magnetic fluid in a non-uniform magnetic field. J. Magn. Magn. Mater. 2018, 447, 116-123. [CrossRef]

18. Braithwaite, D.; Beaugnon, E.; Tournier, R. Magnetically controlled convection in a paramagnetic fluid. Nature 1991, 354, 134-136. [CrossRef]

19. De Vahl Davis, G. Natural convection of air in a square cavity: A bench mark numerical solution. Int. J. Numer. Methods Fluids 1983, 3, 249-264. [CrossRef] 